\title{
Permeability of the Paranodal Junction of Myelinated Nerve Fibers
}

\author{
Amanda Mierzwa, Seema Shroff, and Jack Rosenbluth \\ Department of Physiology and Neuroscience and Rusk Institute, New York University School of Medicine, New York, New York 10016
}

We have used fluorescent dextran tracers to test the tightness of the paranodal junction of living or fixed myelinated fibers in mouse sciatic nerve. Both 3 and $70 \mathrm{kDa}$ tracers are able to penetrate from the perinodal space symmetrically into the paranodes on either side of the node of Ranvier at a rate consistent with diffusion through an elongated helical pathway between the paranodal terminal loops of the myelin sheath. This pathway thus provides an access route for movement of water soluble nutrients and metabolites to and from the internodal axon and constitutes a pathway through which juxtaparanodal potassium channels may be activated and may in turn affect nodal excitability. This pathway may also allow access of antibodies and toxic molecules to the internodal axon in paraneoplastic syndromes and demyelinating diseases.

\section{Introduction}

Early diagrams of myelinated nerve fibers depicted myelin as a tight-fitting lipidic sleeve around the axon, interrupted periodically at nodes of Ranvier (Huxley and Stämpfli, 1949). This architecture appeared not only to insulate the covered axon, but also to prevent short circuiting of nodal action currents under the myelin sheath (Tasaki, 1939). Later studies showed that compact myelin is in fact separated from the axon by a 10-20 nm periaxonal space (Robertson, 1959) except at the paranodal junction (PNJ) flanking each node. There the space narrows to $\sim 2-4 \mathrm{~nm}$ and is traversed by transverse bands, intercellular ridge-like structures that extend between the terminating myelin lamellae and the axolemma (for review, see Rosenbluth, 2009).

The presence of a periaxonal space within the PNJ raises questions about the permeability of the junction. The extent to which the PNJ permits longitudinal current flow is a subject of interest, particularly in view of the accumulations of fast, voltage-gated $\mathrm{K}^{+}$channels in the juxtaparanodal (JP) region of the axolemma under the myelin sheath just beyond the paranode (Wang et al., 1993). Can nodal action currents activate these $\mathrm{K}^{+}$channels? If so, to what extent does their activity affect nodal properties? In view of the high lipid content of compact myelin, additional questions arise about access of the internodal axon to nutrients and other water-soluble molecules (Rosenbluth, 2009).

Previous tracer studies of CNS myelinated fibers showed penetration of colloidal lanthanum into the paranodal junctional cleft (Hirano and Dembitzer, 1969) and microperoxidase reaction product in the internodal periaxonal space (Feder, 1971),

Received Aug. 3, 2010; revised Sept. 13, 2010; accepted Sept. 25, 2010.

This work was supported by research grants from the National Institutes of Health (NS37475) and the National Multiple Sclerosis Society (RG3618). We are indebted to Drs. Charles Nicholson, Sabina Hrabetova, and Robert Thorne for invaluable discussions, to Dr. R. Schiff for help with photography and measurements, and to Chris Petzold for expert technical assistance.

Correspondence should be addressed to Dr. Jack Rosenbluth, Department of Physiology, New York University School of Medicine, 550 First Avenue, PHB 833, New York, NY 10016. E-mail: rosenj03@med.nyu.edu.

DOI:10.1523/JNEUROSCI.4047-10.2010

Copyright $\odot 2010$ the authors $\quad 0270-6474 / 10 / 3015962-07 \$ 15.00 / 0$ suggesting the presence of patent channels through the paranode. In contrast, early electrophysiological studies showed that $\mathrm{K}^{+}$ channel blocking agents had no significant effect on conduction by normal living PNS nerve fibers (Chiu and Ritchie, 1980; Sherratt et al., 1980), implying lack of conductive pathways between $\mathrm{JP} \mathrm{K}^{+}$channels and the node of Ranvier. Equivalent results were obtained with CNS myelinated fibers (Kocsis and Waxman, 1980). Only after manipulation of the fibers, resulting in exposure of the JP K ${ }^{+}$channels, did $\mathrm{K}^{+}$channel block have an effect (Brismar, 1981). More recent in vitro studies, however, have shown that 4-AP (Vabnick et al., 1999) and peptide $\mathrm{K}^{+}$channel blockers (Devaux and Gow, 2008) do modify conduction in small-caliber CNS myelinated fibers.

Based on these disparate results, it is not clear to what extent the PNJ of normal living nerve fibers in vivo is penetrable and to what extent it constitutes a pathway for current flow to and from $\mathrm{JP} \mathrm{K}^{+}$channels and for molecular traffic between the perinodal extracellular space and the internodal periaxonal space.

To assess the tightness of the PNJ in both living and fixed PNS nerve fibers and the extent to which the juxtaparanodal and internodal periaxonal spaces can be accessed through it, we have followed the movement of fluorescent dextran tracers that are relatively inert and nontoxic. These tracers can be applied to nerves in vivo or in vitro, and their location within individual nerve fibers can be determined readily by epifluorescence microscopy.

\section{Materials and Methods}

Live nerves. All animal procedures were performed in accordance with protocols approved by the New York University School of Medicine Institutional Animal Care and Use Committee.

Dextrans (Invitrogen) of molecular weight 3 and $70 \mathrm{kDa}$ coupled to either fluorescein (FITC) or rhodamine B (RB) were reconstituted in $1 \times$ Tris-buffered saline (TBS) $(3 \mathrm{kDa}$-FITC to $10 \mathrm{mg} / \mathrm{ml} ; 70 \mathrm{kDa}-\mathrm{RB}$ to $25 \mathrm{mg} / \mathrm{ml}$ ), aliquotted, and stored at $-80^{\circ} \mathrm{C}$. The $3 \mathrm{kDa}$ dextran used had an estimated diameter of $2.6 \mathrm{~nm}$, and the $70 \mathrm{kDa}$ dextran had an estimated diameter of 12-16 nm (Syková and Nicholson, 2008). At the time of use, aliquots were thawed, mixed together, and diluted $\sim 1: 5$ in $1 \times$ TBS. 
Male or female mice were anesthetized with pentobarbital and the sciatic nerves were exposed in the thigh region. Approximately $10 \mu \mathrm{l}$ of the diluted dextrans was injected into each nerve via a Hamilton syringe and 30 gauge needle, resulting in some swelling and discoloration of the nerve over a length of $\sim 1-2 \mathrm{~cm}$. After injection, the muscles were approximated; the nerve was kept moist, and the animal maintained under anesthesia in a warm chamber for time periods ranging from 0 to $4 \mathrm{~h}$. At the end of that time, the animal was fixed by transcardiac perfusion of $4 \%$ buffered formaldehyde, freshly made up from paraformaldehyde. The injected nerve was simultaneously flooded with fixative and left in situ.

After fixation, the nerve was excised, rinsed six times for 1 min each in $1 \times$ TBS and then teased on a subbed slide with two 31 gauge syringe needles. Teased fibers were mounted in an antifade medium (Vectashield), coverslipped, and examined immediately in an inverted Hoffman interference contrast microscope equipped for epifluorescence microscopy. Total times of exposure to tracer are expressed in minutes as the live time plus the preparation time (prep), e.g., $90(60+30)$.

The teased fibers were surveyed by Hoffman interference contrast microscopy for nodes of Ranvier, appearing as circumferential constrictions in the cylindrical fibers, and those regions were then examined by fluorescence microscopy using filter cubes that provided excitation wave lengths appropriate for FITC-3 kDa dextran and RB-labeled $70 \mathrm{kDa}$ dextran. Fluorescent fibers were photographed through $40 \times$ or $100 \times$ objective lenses, and the Hoffman and fluorescence images were then superimposed. Some fibers were examined by confocal microscopy, and image stacks of longitudinally oriented fibers were rotated to provide end-on views.

Fixed nerves. Mice were anesthetized as described above and the sciatic nerves exposed. The epineurium was incised longitudinally in situ with the tip of a 31 gauge needle, and the nerve was then excised, immersed in buffered 3\%glutaraldehyde/2\%formaldehyde for $2 \mathrm{~d}$, then rinsed and stored in $1 \times$ TBS for up to 1 week. These nerves were then covered with dextran solutions for $0.5-4 \mathrm{~h}$, rinsed, teased, mounted, and examined by Hoffman and epifluorescence microscopy or confocal microscopy to follow tracer penetration in sciatic nerve fibers after fixation. In this paradigm, the time from rinsing to photography of the fluorescent images was $\sim 15 \mathrm{~min}$, and tracer movement during that time is included in the measurements and calculations (e.g., $135 \mathrm{~min}=120+15$ ). All measurements were made on nodes remote from the cut ends of the nerves to exclude tracer entering the fibers through that route.

Measurements of tracer spread. Fluorescence photomicrographs were analyzed using Image software (NIH) to determine the overall extent of longitudinal tracer movement in both directions from each nodal slit. Total spread on both sides was measured, and $0.75 \mu \mathrm{m}$ subtracted to allow for tracer within the nodal gap. The remainder was divided by 2 to obtain average spread into each myelin segment.

The experiments on live nerves entailed a period $(t)$ from 0 to $240 \mathrm{~min}$ after injection of tracer into the sciatic nerve, during which the nerve fibers were exposed to tracer while the mouse was alive and anesthetized, followed by a period of $\sim 30 \mathrm{~min}$ during which the nerve was fixed, excised, rinsed, teased, and mounted for examination. Our evidence from studies of fixed nerves is that aldehyde fixation does not freeze the tracer in place; i.e., the tracer is still able to diffuse during the $30 \mathrm{~min}$ after fixation. Thus, the total time ( $T$ ) for diffusion approximates $t+30 \mathrm{~min}$.

Statistical analysis. Statistical significance was determined with the Student's $t$ test using Microsoft Excel and Statistical Package for the Social Sciences (SPSS). A $p$ value of $<0.05$ was considered significant. A two-tailed $t$ test was used in all cases except for comparing dextran diffusion over time, where we hypothesized an increase in penetration with time, making the one-tailed $t$ test the appropriate statistical tool.

Diffusion calculations. Calculation of root mean square diffusion distances was based on the following relationship: $x^{2}=2 D t$. Values for diffusion coefficient in brain $\left(D^{*}\right)$ in centimeters squared per second were calculated from diffusion coefficients in dilute agarose $(D)$ divided by the square of tortuosity $(\lambda)$ derived from studies of normoxic rat neocortex in vivo (Syková and Nicholson, 2008). The values used in our calculations are as follows: $D^{\star}=5.36 \times 10^{-7}(3 \mathrm{kDa}$ dextran $)$ and $D^{*}=$ $0.648 \times 10^{-7}(70 \mathrm{kDa}$ dextran$)$. Time $(t)$ was expressed in seconds.
To estimate distances diffused at times between those actually measured, we multiplied distance measured at the nearest earlier time point by the square root of the ratio of the respective times.

\section{Results}

Dextran tracer penetration of paranodes in live nerve fibers

After tracer was injected into the sciatic nerve, it first appeared at the node of Ranvier as a fluorescent slit perpendicular to the axonal axis (Fig. $1 A-C$, arrow). The $3 \mathrm{kDa}$ tracer was usually washed out of the nodal slit during the rinse steps, but the $70 \mathrm{kDa}$ tracer often persisted at the node and along the outer surface of the fiber (Fig. $1 E, F$ ). The tracer then penetrated into the fiber on both sides of the nodal slit more or less symmetrically, forming a bar (Fig. $1 E, H$, horizontal line). The extent of penetration varied at any given time point, but by $1.5 \mathrm{~h}$, on average, the abnodal ends of the $3 \mathrm{kDa}$ paranodal bar had split into two tines (Fig. $1 F$, arrows), which then extended beyond into the internode (Fig. $1 G-I$ ), forming hairpin-like configurations (Mierzwa and Rosenbluth, 2006). In all cases, superimposition of interference contrast and fluorescence images showed that tracer extending into the internodal domain still lay within the confines of the fiber, separated from the outermost surface by a narrow, nonfluorescent band (Fig. $1 F, I$ ).

The appearance of the dextran-labeled fibers is variable in the paranodal region. Especially in larger fibers, the margin of the paranodal fluorescence is often ill-defined, and in focal regions it may give rise to radial or oblique extensions. PNS paranodes are well known to display regions in which clusters of terminal loops do not reach the axon surface. At these sites, the periaxonal space widens focally, thus creating periaxonal lacunae in which the tracers can pool, extending barb-like from the axon surface.

Even though the tracers may not have cleanly delineated the paranode because of such irregularities, it is striking that they penetrated rather symmetrically in both abnodal directions from the node. If the spread of tracer resulted from damage to a paranode, it is not likely that both paranodes would be so consistently and so equally damaged as to produce such a multiplicity of double-hairpin configurations around nodes.

To determine the location of the tracers within the fiber, confocal stacks of longitudinally oriented images were rotated electronically to provide end-on views of the nerve fibers. In the $Z$ stack shown in Figure $1 P$, a green fluorescent ring surrounds a dark core. That ring is in turn surrounded by a thick dark ring, and external to that, a faint thin red ring. We interpret these images as representing the axon surrounded by $3 \mathrm{kDa}$ tracer in the periaxonal space, surrounded by compact myelin, surrounded by $70 \mathrm{kDa}$ tracer outside the fiber, probably bound to its basal lamina. The hairpin-like fluorescence seen in longitudinal images presumably arises from the green circumferential ring, which, when viewed from above, results in superimposition of fluorophores near the sides of the ring to a much greater extent than in the frontal view. These images thus correspond to the appearance of a transilluminated empty green bottle, whose edges appear more heavily pigmented even though the glass is of uniform thickness. In the paranodal region, where the axon is much narrower, neither confocal $Z$-stacks nor single-slice orthogonal views were able to resolve the axon per se, presumably because of flare from fluorophores in the surrounding periaxonal space.

The average longitudinal extent of tracer penetration into live fibers increases with time (Table 1; supplemental Fig. S1, available at www.jneurosci.org as supplemental material), progressing from the nodal slit to the paranodal bar and finally the internodal hairpins. The mean measured distances for dextran movement when the tissue was fixed immediately after tracer injection (the $0+30$ time 
point) show the extent of tracer penetration during the $30 \mathrm{~min}$ processing of the tissue. If these tare values $(\sim 1 \mu \mathrm{m}$ for the $3 \mathrm{kDa}$ tracer and $\sim 0.4 \mu \mathrm{m}$ for the $70 \mathrm{kDa}$ tracer) are subtracted from the total distances recorded at later time points, the remainder represents movement only during the period when the nerves were alive.

\section{Dextran penetration of paranodes in fixed fibers}

Equivalent results were obtained from fibers that had been fixed in a mixture of formaldehyde and glutaraldehyde, excised, and soaked in 3 or $70 \mathrm{kDa}$ tracer labeled with fluorescein only (rhodamine B was not used to avoid confusion with glutaraldehyde autofluorescence). The appearance of the 3 $\mathrm{kDa}$ tracer images (Fig. $1 J-L$ ) and $70 \mathrm{kDa}$ tracer images (Fig. 1M-O) corresponded to what is seen after injection of the tracers into live nerves in situ. Here too, the paranodal bar (Fig. $1 K, N$, horizontal line) widened in a shoulder region (Fig. $1 K, N$, arrows) and then formed internodal hairpins (Fig. 1O, arrows) that were clearly seen to lie within the outer margins of the fibers when the fluorescence and interference contrast images were superimposed. For comparable times of exposure to tracer, the glutaraldehyde/formaldehyde-fixed nerves showed penetration to about the same extent as that seen in live, unfixed fibers (Table 2). Since tracer penetration has been observed in both live and fixed fibers, spread of the tracer cannot depend on endocytosis or intracellular transport or other energydependent cellular processes. The simplest mechanism to account for our results is, therefore, passive diffusion of these relatively inert tracers in both fixed and live nerve fibers.

\section{Proportion of fibers showing paranodal penetration}

In live-injected and in fixed sciatic nerves, both 3 and $70 \mathrm{kDa}$ dextrans showed measurable penetration into some of the paranodes (hits) at each time point studied. The number of paranodes penetrated in each coverslip studied is, however, very different for the respective tracers. As shown in supplemental Table S2, available at www. jneurosci.org as supplemental material, the $3 \mathrm{kDa}$ tracer penetrated $>20 \times$ as many paranodes as the $70 \mathrm{kDa}$ tracer did in fixed nerves. In live-injected fibers, the difference is smaller, but the trend is similar.

\section{Dextran tracer penetration of Schmidt-Lanterman clefts}

In live-injected nerves, most fibers showed no penetration of tracers except, as described above, via the paranodal region. However, occasional fibers (Fig. $2 A-C$ ) showed penetration in internodal regions in a configuration consistent with movement through conical Schmidt-Lanterman (SL) clefts extending ob-
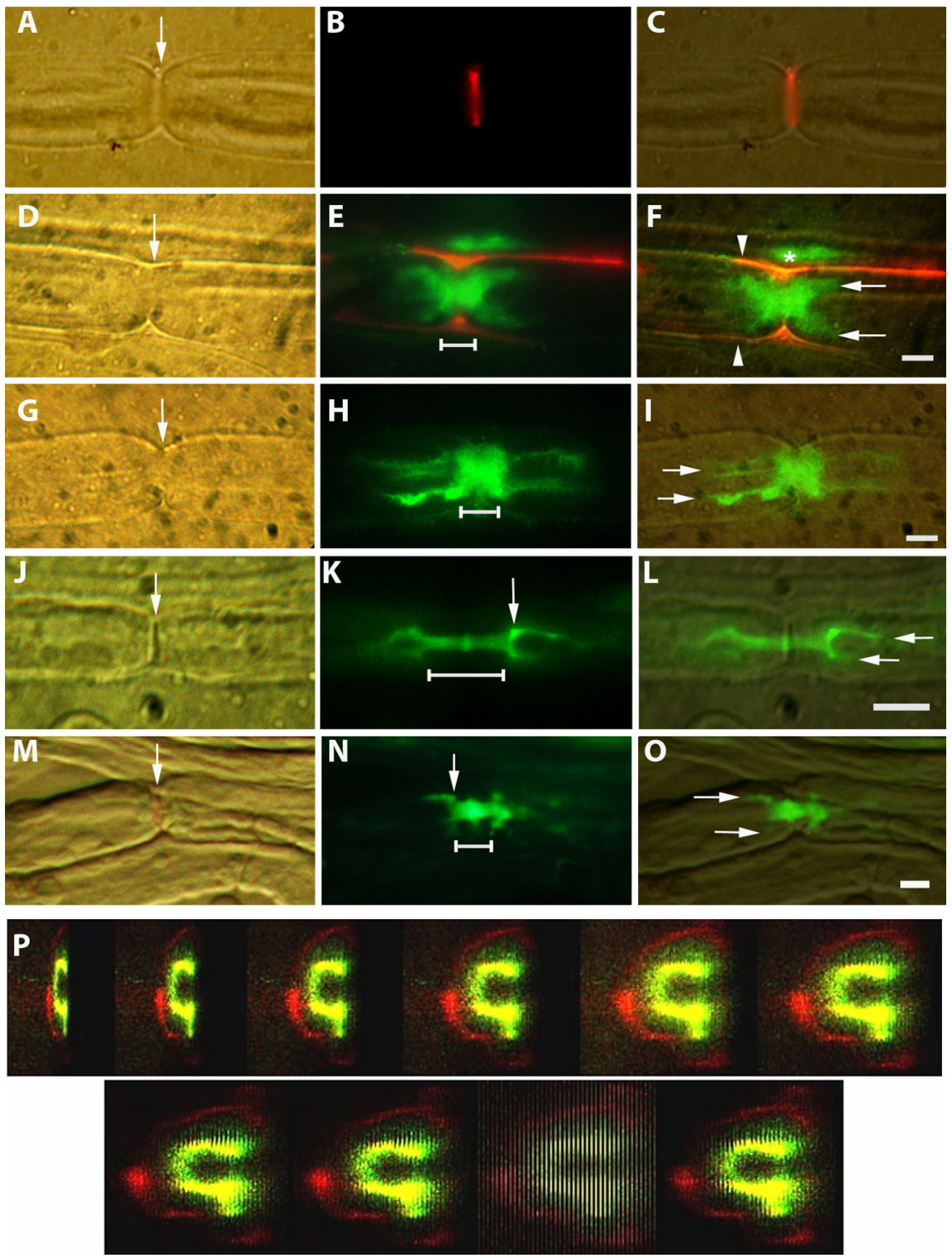

Figure 1. $\quad \boldsymbol{A}-\mathbf{0}$, Hoffman, fluorescence, and merged images showing penetration of dextran tracers into node/paranode regions of sciatic nerve myelinated fibers. Scale bars, $5 \mu \mathrm{m}$. The node of Ranvier is indicated by arrows in $\boldsymbol{A}, \boldsymbol{D}, \boldsymbol{G}, \boldsymbol{J}$, and $\boldsymbol{M}$. The linear extent of paranodal bars is indicated by horizontal lines in $\boldsymbol{E}, \boldsymbol{H}, \boldsymbol{K}$, and $\boldsymbol{N}$. $\boldsymbol{A}$-C, Live injection. $70 \mathrm{kDa}$ tracer (red) fills the nodal slit (arrow). $\boldsymbol{D}-\boldsymbol{F}$, Live injection. $70 \mathrm{kDa}$ tracer (red) outlines a nerve fiber $(\boldsymbol{E}, \boldsymbol{F})$. $\boldsymbol{F}$, Between the red outlines (arrowheads), $3 \mathrm{kDa}$ tracer (green) has penetrated from the node into the fiber symmetrically forming a thick paranodal bar just beginning to give rise to the tines (arrows) of hairpins at both Some $3 \mathrm{kDa}$ tracer above this fiber $\left(^{*}\right)$ has been trapped between it and a second fiber whose outline is visible above. $\mathbf{G}-\mathbf{I}$, Live njection. $3 \mathrm{kDa}$ tracer (green) forms a paranodal bar, which widens to form conspicuous hairpins (arrows) symmetrically on both sides of he node. $\boldsymbol{J}-\boldsymbol{L}$, Fixed nerve exposed to $3 \mathrm{kDa}$ tracer (green), which forms a narrow paranodal bar $(\boldsymbol{K}$, line) that is slightly wider just at the , both ends, the bar widens in a shoulder region ( $\boldsymbol{K}$, arrow), where it forms hairpins that extend into the internodal periaxonal space. $\mathbf{M}-\mathbf{0}$, Fixed nerve exposed to $70 \mathrm{kDa}$ tracer (green), which forms a short bar ( $\boldsymbol{N}$, line) that gives rise to square shoulder regions $(\boldsymbol{N}$, arrow) t each end and the beginnings of hairpin tines $(\boldsymbol{O}$, arrows). $\boldsymbol{P}$, Live injection. Confocal Z-stack rotated to show dark core (internodal axon) surrounded by $3 \mathrm{kDa}$ dextran (green) in the periaxonal space. That in turn is surrounded by a dark ring (compactmyelin) and outside of that a thin red ring (70 kDa dextran) along the outside of the fiber. The ninth image in the series shows the confocal slices end on.

liquely across the sheath on either side. The same fiber often showed multiple clefts along its length, even though surrounded by other fibers that showed none. In the example illustrated in Figure $2 B$, the $3 \mathrm{kDa}$ tracer extended across the full thickness of the myelin sheath into the periaxonal space and then spread in both directions along the surface of the axon. The $70 \mathrm{kDa}$ tracer (Fig. $2 \mathrm{~A}$ ) filled the cleft but did not spread within the periaxonal space.

In nerves soaked in the tracers after fixation, SL clefts (Fig. $2 D$, arrow) also showed dextran penetration equivalent to that seen in 
Table 1. Dextran penetration (in $\mu \mathrm{m}$ ) in live-injected fibers

\begin{tabular}{lllllll}
\hline \multirow{2}{*}{ Tracer } & Time $(\mathrm{min})$ & \multicolumn{3}{l}{ Calculated } & & \\
\cline { 3 - 5 } & total (live + prep$)$ & 1 & 2 & 3 & Measured & $n$ \\
\hline $3 \mathrm{kDa}$ & $30(0+30)$ & 440 & 409 & 2.8 & $1.0 \pm 0.9$ & 14 \\
& $60(30+30)$ & 621 & 590 & 4.0 & $3.2 \pm 2.8$ & 16 \\
$70 \mathrm{kDa}$ & $90(60+30)$ & 762 & 731 & 4.9 & $5.4 \pm 4.2$ & 45 \\
& $30(0+30)$ & 153 & 122 & 1.0 & $0.4 \pm 0.3$ & 14 \\
& $60(30+30)$ & 216 & 185 & 1.4 & $1.3 \pm 0.9$ & 16 \\
& $90(60+30)$ & 264 & 233 & 1.7 & $1.8 \pm 1.3$ & 45 \\
& $150(120+30)$ & 341 & 310 & 2.2 & $5.9 \pm 2.8$ & 24 \\
\hline
\end{tabular}

Calculated and measured dextran tracer penetration in live fibers. Tracer penetration through pathways 1, 2, and 3 in a fiber assumed to have a paranode $5 \mu \mathrm{m}$ in diameter and $5 \mu \mathrm{m}$ long with transverse bands oriented at $8^{\circ}$ to the transverse plane and with paranodal loops spaced at $0.1 \mu \mathrm{m}$ intervals. Average measured penetration approximates calculated penetration through pathway 3 but differs from that through pathways 1 or 2 by approximately two orders of magnitude. Each calculated value represents the axial distance $(\mu \mathrm{m})$ moved on one side of a node in the paranode plus distance in the internode. Each Measured value represents apparent axial extent of tracer penetration $( \pm S D) . n$, Number of paranodes measured.

Table 2. Dextran penetration (in $\mu \mathrm{m}$ ) in fixed fibers

\begin{tabular}{lcllllll}
\hline \multirow{2}{*}{ Tracer } & $\begin{array}{l}\text { Time } \\
(\mathrm{min})\end{array}$ & 1 & 2 & 3 & Measured & $n$ & Estimated \\
\cline { 3 - 6 } $3 \mathrm{kDa}$ & 30 & 440 & 409 & 2.8 & $2.2 \pm 1.4$ & 15 & \\
& 45 & 540 & 509 & 3.4 & $2.6 \pm 1.5$ & 35 & \\
& 60 & 621 & 590 & 4.0 & & & 3.0 \\
$70 \mathrm{kDa}$ & 75 & 650 & 619 & 4.1 & $5.3 \pm 2.8$ & 210 & \\
& 135 & 324 & 293 & 2.1 & $4.4 \pm 2.6$ & 207 & \\
& 150 & 342 & 311 & 2.2 & & & 4.6
\end{tabular}

Calculated and measured dextran penetration in fixed fibers. The expected tracer penetration through different pathways in a fiber was calculated as in Table 1. Here too, average measured penetration at all time points approximates calculated penetration through pathway 3 , but differs from that through pathways 1 and 2 by approximately two orders of magnitude. For comparison with live fibers, diffusion for corresponding time points has been estimated from the measured values. $n$, Number of paranodes measured.

live nerves (Fig. 2E,F). Thus, here too, the penetration cannot be attributed to endocytosis or any other energy-dependent process and therefore must occur by passive diffusion.

\section{Distance penetrated by tracers}

Calculation of diffusion distances, based on the sizes of the tracers and the diffusion coefficients obtained from prior studies of tracer spread in live brain (Syková and Nicholson, 2008), showed that the $3 \mathrm{kDa}$ tracer would be expected to move $440 \mu \mathrm{m}$ in 30 min. This figure, based on root mean square calculations, depends on detection sensitivity, tortuosity, and other factors and is, therefore, an approximation. Nevertheless, the distances actually seen in live and fixed preparations ( 0.9 and $2.2 \mu \mathrm{m})$ were not even close to those calculated, differing, rather, by more than two orders of magnitude. Similarly, the $70 \mathrm{kDa}$ tracer would be expected to spread $324 \mu \mathrm{m}$ in $135 \mathrm{~min}$ but was seen to penetrate only $4.4 \mu \mathrm{m}$, again nearly two orders of magnitude less. Thus, the average measured distances were far below those predicted.

Tables 1 and 2 show average observed distances of tracer movement at various time points in live and fixed fibers, respectively. In all cases, there is a marked discrepancy between calculated diffusion distances and the penetration actually seen. This could reflect impeded diffusion due to friction between the tracer and the walls of the diffusion pathway, an effect that should be greater for the larger tracer. However, the apparent slowing of diffusion was comparable for both of the tracers used, despite the difference in their size. The discrepancy could also reflect increased viscosity of the fluid in the diffusion pathway. But in the fixed nerves, the composition of the tissue spaces is undoubtedly different from that in the living nerves; yet the observed movement was comparable in the live and fixed nerves. In view of these considerations, the simplest explanation for
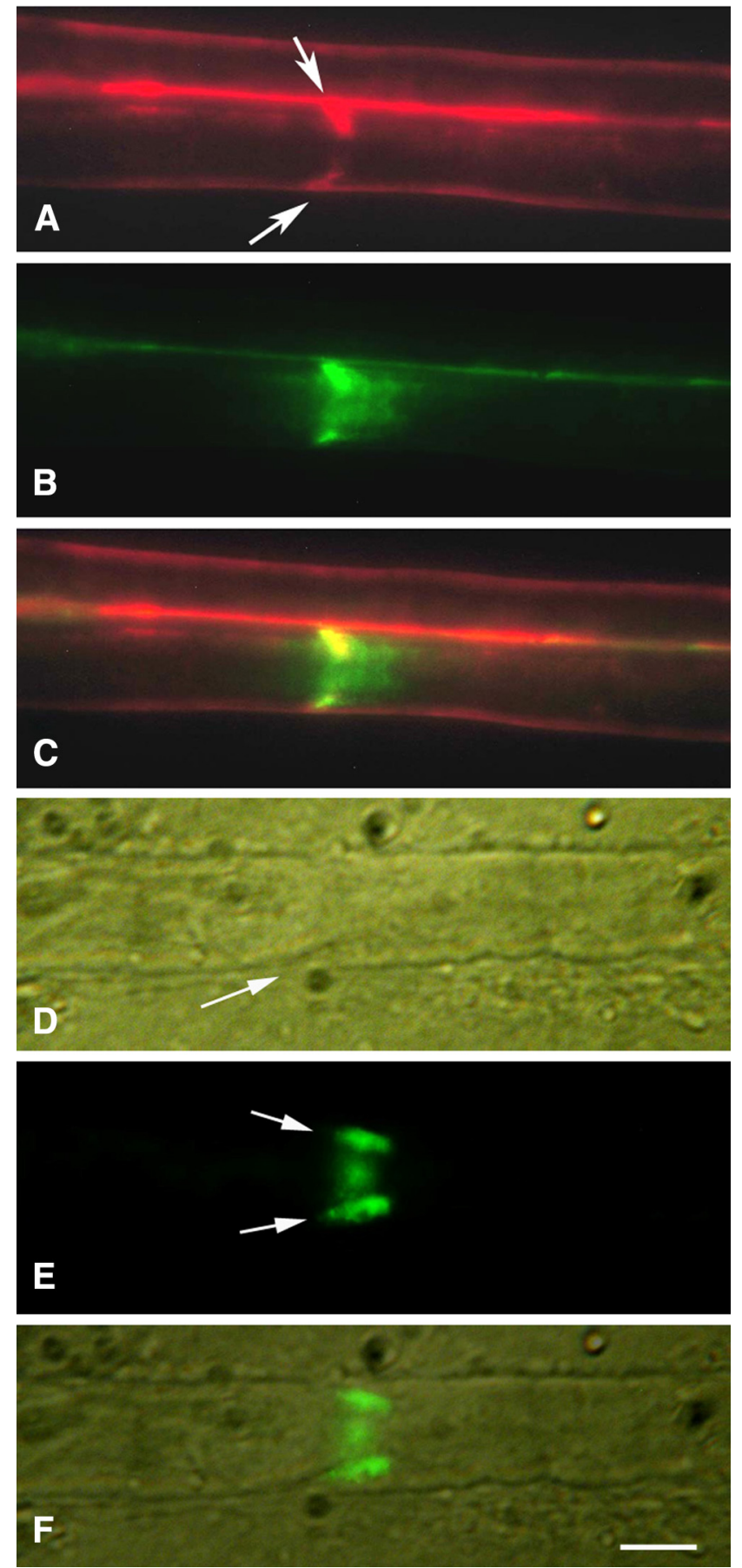

Figure 2. $\quad \boldsymbol{A}-\boldsymbol{C}$, Schmidt-Lanterman cleft (live injection). Both tracers, $70 \mathrm{kDa}$ (red) and $3 \mathrm{kDa}$ (green), outline the outer surface of the fiber and both extend obliquely from the fiber surface toward the axon (arrows). The $3 \mathrm{kDa}$ tracer then spreads longitudinally along the axon in both directions $(\boldsymbol{B})$. The $70 \mathrm{kDa}$ tracer $(\boldsymbol{A})$ does not extend beyond the inner end of the cleft. $\boldsymbol{C}$, Merged $\boldsymbol{A}$ and $\boldsymbol{B}$ images. In $\boldsymbol{A}$ and $\boldsymbol{C}$, the red outline of a second fiber is visible above the outline of the first. $\boldsymbol{D}-\boldsymbol{F}$, SchmidtLanterman cleft in fixed fiber exposed to $70 \mathrm{kDa}$ dextran (green) for $4 \mathrm{~h}$. Arrows in $\boldsymbol{E}$ show the tracer extending obliquely into the cleft $(\boldsymbol{D}$, arrow) toward the axon from both sides of the fiber and visible also in the frontal view between. $\boldsymbol{F}$, Merged $\boldsymbol{D}$ and $\boldsymbol{E}$ images. Scale bar, $5 \mu \mathrm{m}$.

our observations is that the diffusion pathway within the nerve fibers is much longer than it appears to be.

\section{Pathways through the paranode}

Extracellular spread of the tracers symmetrically within the nerve fiber on either side of the node could take place through the paranode, as described below and illustrated in Figure 3. 
Pathway 1

Dextran could diffuse axially within the $2-4 \mathrm{~nm}$ paranodal junctional gap right across the transverse bands to reach the juxtaparanode and then beyond within the internodal periaxonal space. The pathway through the paranode in this case would consist of a cylindrical space $\sim 2-4 \mathrm{~nm}$ thick and several micrometers long, corresponding to the length of the paranode.

\section{Pathway 2}

Dextran could diffuse along the spaces between the ridge-like transverse bands, as suggested by Hirano and Dembitzer (1969). In this case, since the transverse bands are oriented obliquely with respect to fiber axis, the tracers, instead of moving axially, would move through the PNJ at a markedly oblique angle, approximating, in mice, $8^{\circ}$ off the plane transverse to the axonal axis (Rosenbluth, 2009). The length of this path would be inversely proportional to the sine of that angle or approximately sevenfold longer than pathway 1 , which passes axially across the PNJ.

\section{The third pathway}

As indicated above, however, neither of these pathways can account for the relatively short apparent distance moved by the tracers, resulting in a disparity between measured and calculated diffusion distances approximating two orders of magnitude. Moreover, both of these pathways are only 2-4 $\mathrm{nm}$ in width. Although the $3 \mathrm{kDa}$ tracer is $\sim 2.6 \mathrm{~nm}$ in diameter, i.e., comparable to the apparent width of the paranodal periaxonal space, the $70 \mathrm{kDa}$ tracer, $\sim 12-16 \mathrm{~nm}$ in diameter, is larger than that by several fold. For this reason, passive diffusion through the paranodal periaxonal space, either axially (pathway 1) or obliquely (pathway 2), seems impossible for the $70 \mathrm{kDa}$ tracer and unlikely for the $3 \mathrm{kDa}$ tracer. To account for the observed distances, we must therefore postulate a different route.

Myelin is well known to form by spiral wrapping of the myelin-forming cell around the axon. The resulting PNJs at the edges of each myelin segment are, accordingly, helical in form (Robertson, 1959). Thus, each paranodal loop seen in longitudinal sections is in fact part of a single continuous cytoplasmic column sectioned multiple times as it winds around the axonal circumference. Adjacent loops, spaced $\sim 0.1 \mu \mathrm{m}$ apart in the axial direction, appose each other closely and indeed form tight junctions with each other, but near the axonal surface their plasma membranes curve away from each other, resulting in the formation of a small extracellular space, roughly triangular in profile, between adjacent loops close to their junction with the axolemma. Just as the loops themselves are part of a continuous helical cytoplasmic column, the triangular spaces between them form a continuous extracellular channel parallel to the cytoplasmic column (Fig. 3), extending helically with a pitch of $\sim 0.1 \mu \mathrm{m}$ from the node of Ranvier through the paranode to the juxtaparanodal periaxonal space. This narrow channel is significantly wider than the paranodal junctional cleft and thus represents a pathway through the paranode of sufficient caliber to accommodate the dextran tracers we used. This pathway is, however, much longer than the length of the paranode.

Specifically, each turn of this extracellular helix is approximately as long as the axonal circumference, and the number of turns of the helix depends on the number of myelin lamellae that form terminal loops against the axon. Thus, if an axon were 10 $\mu \mathrm{m}$ in diameter, narrowing to $5 \mu \mathrm{m}$ in the paranodal region, with a 5 - $\mu \mathrm{m}$-long paranode comprised of 50 paranodal loops having a helical pitch of $\sim 0.1 \mu \mathrm{m}$, the length of the helix from the node of Ranvier to the juxtaparanodal region would be approximately

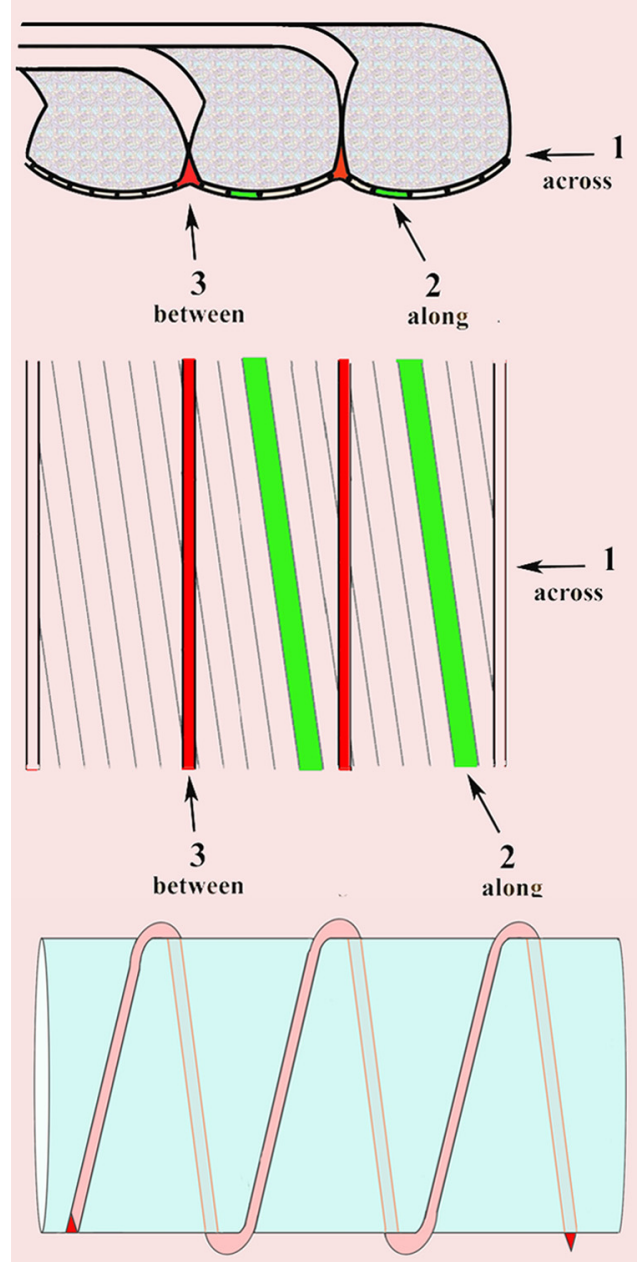

Figure 3. Schematic representations of possible pathways of diffusion. Top, Side view of paranodal loops. Middle, Tangential view. Pathway 1 goes through the junctional cleft across the transverse bands. Pathway 2 also goes through the junctional cleft but passes along the transverse bands at $\sim 8$ degrees from transverse. Pathway 3 does not go through the junctional cleft but passes between paranodal loops. Bottom, Longitudinal view of pathway 3 winding helically around an axon.

$5 \pi \times 50$, or $\sim 785 \mu \mathrm{m}$. In contrast, pathway 1, passing axially through the paranodal periaxonal space, would be only $\sim 5 \mu \mathrm{m}$ long, and pathway 2, passing obliquely along the transverse bands, would be only $\sim 35 \mu \mathrm{m}$ long. A fluorescent particle following the third pathway would have to move nearly $800 \mu \mathrm{m}$ helically to advance $5 \mu \mathrm{m}$ in the axial direction. Beyond the paranode, the particle would move within the internodal periaxonal space directly in the axial direction at a much greater apparent rate.

Comparing the calculated distances diffused through these three pathways with the apparent distances moved in the axial direction (Tables 1,2), it is clear that only movement through the third pathway can account for the observed progression of the dextran tracers through the paranode; extracellular pathways 1 and 2 are far too short to account for the data, as the intracellular diffusion path through the axon would be in the event that some tracer had crossed the nodal axolemma. Correspondingly, the calculated diffusion time for the $3 \mathrm{kDa}$ tracer to traverse the paranode axially is on the order of seconds in pathways $1(0.23 \mathrm{~s})$ and 2 (12 s), but the observed time is on the order of hours ( $1.5 \mathrm{~h})$, again consistent with the movement of dextran through the circuitous pathway 3 (calculated time to traverse the $5 \mu \mathrm{m}$ paranode, $\sim 95 \mathrm{~min})$. 
That the $70 \mathrm{kDa}$ tracer follows the helical pathway is expected, as discussed above, considering the size of this tracer, which is several times larger than the width of the PNJ cleft. Surprisingly, the diffusion calculations indicate that the $3 \mathrm{kDa}$ tracer also follows this pathway. Even in a mouse mutant (CST-null) characterized by a gross deficiency of transverse bands, the permeability of the PNJ to $3 \mathrm{kDa}$ dextran is not increased over that in control mice. Thus, here too the tracer apparently follows pathway 3 through the paranode rather than pathways 1 or 2 through the junctional cleft (Shroff et al., 2009).

Diffusion beyond the paranode is complicated by the fact that the cross-sectional area of the internodal periaxonal space is much larger than that of the helical pathway 3. Specifically, if the cross-section of pathway 3 approximates an equilateral triangle $20 \mathrm{~nm}$ on each side, the area of the triangle would approximate $170 \mathrm{~nm}^{2}$. Pathway 3 would empty into the periaxonal space of the juxtaparanode, whose diameter, using the example above, would then quickly widen to $10 \mu \mathrm{m}$ in the internodal region. The crosssectional area of the internodal periaxonal space would thus approximate the circumference of the axon multiplied by the width of the space, i.e., $10 \pi \times 10^{3} \mathrm{~nm} \times 10-20 \mathrm{~nm}$, or $\sim 300,000-600,000$ $\mathrm{nm}^{2}$, a difference of $\sim 1800-3500$-fold. Tracer following the third pathway would thus become markedly diluted and, as the tracer continued to diffuse axially along the internode, it would be replenished at a very limited rate via the efflux from pathway 3 . For these reasons, tracer moving beyond the paranode may rapidly diminish in concentration below the level of detection, and therefore measurements in this region may not be accurate. This probably accounts for the small apparent difference in $3 \mathrm{kDa}$ tracer penetration at 150 and $270 \mathrm{~min}$ (supplemental Fig. S1, available at www. jneurosci.org as supplemental material). Despite the dilution factor, the $3 \mathrm{kDa}$ tracer does, nevertheless, become visible in the juxtaparanode and in internodal hairpins at a time consistent with diffusion through pathway 3 in the paranode.

\section{Discussion}

\section{Functional significance of paranodal permeability}

Penetration of the paranodal junction by water-soluble molecules provides a means by which a variety of biologically important materials can reach the internodal axon. In view of the high lipid content of myelin, aqueous solutes cannot diffuse across the myelin sheath to the periaxonal space. Here we provide evidence for a paranodal pathway bypassing this barrier and, to a limited degree, a pathway through SL clefts. These paths would permit such materials as glucose, other water-soluble nutrients, and hormones such as thyroxin and insulin to diffuse into the internodal periaxonal space, forming a reservoir there in immediate contact with the axolemma all along its length. These paths also represent routes for diffusion of watersoluble metabolites away from the axon.

Our results are relevant also to human diseases associated with penetration of peripheral nerve myelin sheaths by antibodies or toxic agents. Guillain-Barre syndrome, an autoimmune disease in which components of myelin and/or axons are damaged by a cellular or humoral attack, may depend on penetration of the PNJ by antibodies or such immune effectors as granzyme and complement components, as well as by cell processes. Our results are relevant also to acquired neuromyotonia, which has been shown to result from autoantibodies to $\mathrm{JP} \mathrm{K}^{+}$channels (Kleopa et al., 2006). Similar mechanisms may operate in paraneoplastic neuropathies (Vincent, 2004). Our permeability data may also be relevant to CNS diseases such as multiple sclerosis and central paraneoplastic syndromes. Small fluorescent peptides have been shown to reach and bind to $\mathrm{JP} \mathrm{K}^{+}$channels in CNS explants (Devaux and Gow, 2008). The permeability of living CNS para- nodal junctions to larger macromolecules, the size of antibodies, is not yet known, however.

Finally, demonstration of a patent pathway through the paranode opens up the prospect that under some circumstances enough current could follow that route to activate JP $\mathrm{K}^{+}$channels and that the latter could reciprocally affect nodal excitability. These JP K ${ }^{+}$channels have been a source of consternation because they are covered by the myelin sheath and apparently isolated from the node and thus have no obvious role in signal propagation. Yet the nerve cells make a considerable investment in producing them and transporting them to their ultimate location, and their absence does have functional consequences (Chiu et al., 1999). The pathway through the paranode demonstrated here renders them accessible to a limited and perhaps variable degree, depending on the physiologic (Morán and Mateu, 1983) or developmental (Vabnick et al., 1999) state of the nerve and any pathologic processes operating, and thus represents a route by which these apparently isolated channels may affect nodal properties and behavior. This route for current flow, which we show here is patent in living nerve fibers, is in addition to other possible extracellular routes for current flow, e.g., pathways 1 and 2 through the PNJ and the extracellular spaces in SL clefts, whose conductances are unknown.

The evidence that JP $\mathrm{K}^{+}$channels affect nodal behavior in normal CNS myelinated fibers, particularly those of small caliber (Devaux and Gow, 2008), is consistent with our suggestion that pathway 3 might serve as a route through which $\mathrm{K}^{+}$channel activity affects nodal behavior. The length of that path depends on the number of helical wraps made by the paranodal edges of the sheath, which in turn depends on the number of myelin lamellae. Thus, in the example of the PNS fiber referred to earlier with $5 \mu \mathrm{m}$ paranodes and 50 lamellae of myelin, pathway 3 would be very long-785 $\mu \mathrm{m}$. In a CNS fiber with $1 \mu \mathrm{m}$ diameter paranodes and 15 myelin lamellae, pathway 3 would be only $47 \mu \mathrm{m}$, a 16-fold reduction. Thus, in small-caliber fibers, $\mathrm{JP} \mathrm{K}^{+}$channels are connected to the node by a much shorter and correspondingly less resistive pathway than that of large-caliber fibers and are therefore more likely to exert effects on nodal behavior. To what extent the results of the in vitro studies cited above reflect the behavior of small-caliber myelinated fibers in vivo is uncertain.

Evidence that access to and from $\mathrm{JP} \mathrm{K}^{+}$channels affects saltatory conduction also comes from studies of dysmyelinating mutants lacking transverse bands, e.g., the Caspr-null mouse, which display a pronounced reduction in PNS conduction velocity (Bhat et al., 2001). In the absence of transverse bands, these channels are mislocated to the paranode, and the path interconnecting them with the node is thus greatly shortened. In contrast, dysmyelinating mutants whose transverse bands are preserved show normal segregation of $\mathrm{K}^{+}$channels to the juxtaparanode, and in those, normal conduction velocity is preserved (Mierzwa et al., 2010).

\section{SL clefts}

These structures have been regarded as possibly artifacts of specimen preparation or shearing defects (Robertson, 1958), but studies of living nerve fibers in culture showed SL clefts to be present there too (Bunge et al., 1967). It has also been suggested that they are dynamic; i.e., they can translocate along the axon, or they can open and close (Hall and Williams, 1970).

In all cases, SL clefts are characterized by two features (Robertson, 1958; Hall and Williams, 1970), as follows: (1) a conical stack of myelin lamellae that lose their compaction over a short length; i.e., each major dense line, representing the fused cytoplasmic surfaces of the myelinating Schwann cell, splits to enclose 
cytoplasm; and (2) the external surfaces of the apposed Schwann cell membranes, which form the intraperiod (or intermediate) line, separate to create a larger aqueous gap $\sim 10 \mathrm{~nm}$ in width, which may expand even further under some circumstances, e.g., a hypo-osmotic environment. Circumferential microtubules have been described within the cytoplasmic component, and both adhesive and tight junctions have been described linking the component membrane pairs together (Hall and Williams, 1970).

Nodes of Ranvier, where the external contour of the nerve fiber changes dramatically, are readily identified by Hoffman interference contrast microscopy. SL clefts, in contrast, where the axon diameter is unchanged, are much more difficult to recognize and could be identified only infrequently in the Hoffman mode in our teased preparations. Hence, although we know that tracer was able to penetrate some, as shown in Figure 2, we cannot say what proportion of the total that represents. Biochemically, myelin-associated glycoprotein is associated with regions of cytoplasm-containing myelin membranes, including the SL clefts (Schober et al., 1981; Trapp, 1990), along with Nectin-like proteins (Maurel et al., 2007). A circumferential strip of axolemma containing Caspr, flanked by shaker-type $\mathrm{K}^{+}$channels, faces the innermost extent of each SL cleft cone (Arroyo et al., 1999). Considering the complexity of the membrane differentiations associated with SL clefts, it is difficult to imagine that this constellation of structures could be rapidly mobile within the myelin sheath. However, in the absence of spacers, comparable to transverse bands, to maintain a constant separation between the apposed external surfaces of the SL cleft membranes, variations in gap width depending on functional state or environment might well occur and might account for the variability we see in penetration of SL clefts by dextran tracers. Thus, SL clefts may be subject to much greater fluctuation in permeability and conductance than paranodal junctions are.

\section{Summary and conclusions}

We show that 3 and $70 \mathrm{kDa}$ dextran tracers are able to penetrate through the paranodes of myelinated nerve fibers of mouse sciatic nerve both in life and after fixation. Thus, a patent pathway through the paranode that can accommodate molecules as large as $\sim 12-16 \mathrm{~nm}$ in diameter exists in living fibers and is preserved after fixation. Since movement of the tracers takes place at about the same rate in live and fixed nerves; the movement cannot depend on endocytosis or other metabolism-dependent processes and is most simply explained by passive diffusion.

The rate of movement of both 3 and $70 \mathrm{kDa}$ dextrans through the paranodes is consistent with movement through an elongated pathway, which we propose consists of the helical channel we refer to as pathway 3. This channel passes between successive terminal loops of the paranodal junction beginning at the perinodal space and ending under the myelin sheath at the juxtaparanodal periaxonal space.

We propose also that this pathway serves as a route for movement of water-soluble materials to and from the internodal periaxonal space and that it may serve as a pathway for current flow to and from juxtaparanodal $\mathrm{K}^{+}$channels.

\section{References}

Arroyo EJ, Xu YT, Zhou L, Messing A, Peles E, Chiu SY, Scherer SS (1999) Myelinating Schwann cells determine the internodal localization of Kv1.1, Kv1.2, Kvbeta2, and Caspr. J Neurocytol 28:333-347.

Bhat MA, Rios JC, Lu Y, Garcia-Fresco GP, Ching W, St Martin M, Li J, Einheber S, Chesler M, Rosenbluth J, Salzer JL, Bellen HJ (2001) Axonglia interactions and the domain organization of myelinated axons require neurexin IV/Caspr/paranodin. Neuron 30:369-383.
Brismar T (1981) Specific permeability properties of demyelinated rat nerve fibres. Acta Physiol Scand 113:167-176.

Bunge MB, Bunge RP, Peterson ER, Murray MR (1967) A light and electron microscope study of long-term organized cultures of rat dorsal root ganglia. J Cell Biol 32:439-466.

Chiu SY, Ritchie JM (1980) Potassium channels in nodal and internodal axonal membrane of mammalian myelinated fibres. Nature 284:170-171.

Chiu SY, Zhou L, Zhang CL, Messing A (1999) Analysis of potassium channel functions in mammalian axons by gene knockouts. J Neurocytol 28:349-364.

Devaux J, Gow A (2008) Tight junctions potentiate the insulative properties of small CNS myelinated axons. J Cell Biol 183:909-921.

Feder N (1971) Microperoxidase: an ultrastructural tracer of low molecular weight. J Cell Biol 51:339-343.

Hall SM, Williams PL (1970) Studies on the "incisures" of Schmidt and Lanterman. J Cell Sci 6:767-791.

Hirano A, Dembitzer HM (1969) The transverse bands as a means of access to the periaxonal space of the central myelinated fibre. J Ultrastruct Res 28:141-149.

Huxley AF, Stämpfli R (1949) Evidence for saltatory conduction in peripheral myelinated nerve-fibers. J Physiol 108:315-339.

Kleopa KA, Elman LB, Lang B, Vincent A, Scherer SS (2006) Neuromyotonia and limbic encephalitis sera target mature Shaker-type K+ channels: subunit specificity correlates with clinical manifestations. Brain 129:1570-1584.

Kocsis JD, Waxman SG (1980) Absence of potassium conductance in central myelinated axons. Nature 287:348-349.

Maurel P, Einheber S, Galinska J, Thaker P, Lam I, Rubin MB, Scherer SS, Murakami Y, Gutmann DH, Salzer JL (2007) Nectin-like proteins mediate axon Schwann cell interactions along the internode and are essential for myelination. J Cell Biol 178:861-874.

Mierzwa AJ, Rosenbluth J (2006) Lateral permeability of the paranodal junction in myelinated nerve fibers. Soc Neurosci Abstr 32:638.6.

Mierzwa AJ, Arevalo JC, Schiff R, Chao MV, Rosenbluth J (2010) Role of transverse bands in maintaining paranodal structure and axolemmal domain organization in myelinated nerve fibers: effect on longevity in dysmyelinated mutant mice. J Comp Neurol 518:2841-2853.

Morán O, Mateu L (1983) Loosening of paranodal myelin by repetitive propagation of action potentials. Nature 304:344-345.

Robertson JD (1958) The ultrastructure of Schmidt-Lanterman clefts and related shearing defects of the myelin sheath. J Biophys Biochem Cytol 4:39-46.

Robertson JD (1959) Preliminary observations on the ultrastructure of nodes of Ranvier. Z Zellforsch Mikrosk Anat 50:553-560.

Rosenbluth J (2009) Multiple functions of the paranodal junction of myelinated nerve fibers. J Neurosci Res 87:3250-3258.

Schober R, Itoyama Y, Sternberger NH, Trapp BD, Richardson EP, Asbury AK, Quarles RH, Webster HD (1981) Immunocytochemical study of P0 glycoprotein, P1 and P2 basic proteins, and myelin-associated glycoprotein (MAG) in lesions of idiopathic polyneuritis. Neuropathol Appl Neurobiol 7:421-434.

Sherratt RM, Bostock H, Sears TA (1980) Effects of 4-aminopyridine on normal and demyelinated mammalian nerve fibres. Nature 283:570 -572 .

Shroff S, Mierzwa A, Rosenbluth J (2009) Permeability of the paranodal junction to dextran tracers in CST-null and wild-type sciatic nerves. Soc Neurosci Abstr 35:724.14

Syková E, Nicholson C (2008) Diffusion in brain extracellular space. Physiol Rev 88:1277-1340.

Tasaki I (1939) The electro-saltatory transmission of the nerve impulse and the effect of narcosis on the nerve fiber. Am J Physiol 127:211-227.

Trapp BD (1990) The myelin-associated glycoprotein: location and potential functions. In Myelination and dysmyelination, Vol. 605 (Colman D, Duncan I, Skoff R, eds), pp. 29-43. New York: The New York Academy of Sciences.

Vabnick I, Trimmer JS, Schwarz TL, Levinson SR, Risal D, Shrager P (1999) Dynamic potassium channel distributions during axonal development prevent aberrant firing patterns. J Neurosci 19:747-758.

Vincent A (2004) Antibody-mediated disorders of neuromuscular transmission. Suppl Clin Neurophysiol 57:147-158.

Wang H, Kunkel DD, Martin TM, Schwartzkroin PA, Tempel BL (1993) Heteromultimeric $\mathrm{K}+$ channels in terminal and juxtaparanodal regions of axons. Nature 365:75-79. 